\section{Waste-disposal chute}

The correct disposal of waste is essential for daily life, and this applies at the cellular level too. Misfolded proteins in the endoplasmic reticulum (ER) are retro-translocated (or dislocated) to the cytosol for degradation, and blocking this pathway causes ER stress. Some components of the pathway are known - for example, the cytosolic ATPase p97 probably 'pulls' misfolded proteins out of the ER. However, the channel that mediates retro-translocation has been a matter of speculation. Two papers in Nature, though, now identify a protein that might form such a channel.

In the first paper, Lilley and Ploegh studied the US11 protein of the human cytomegalovirus, which targets the major histocompatibility class-I heavy chain for retro-translocation. A Q192L mutant of US11 cannot maintain this retro-translocation, and the presence of this glutamine residue in the transmembrane region of US11 indicated that it might interact with host proteins.

The authors therefore developed an affinity-purification approach to examine which cellular proteins bind to US11 versus US11 $1^{\mathrm{Q} 192 \mathrm{~L}}$, and they identified a protein — which was named Derlin-1 — that specifically associated with US11. This transmembrane protein is a homologue of Saccharomyces cerevisiae Der1, which is required for the degradation of a subset of misfolded ER proteins.

Using antibodies against Derlin-1 in immunoprecipitation experiments, Lilley and Ploegh confirmed the Derlin-1-US11 association and showed that the class-I heavy chain was also recovered with Derlin-1. This was not the case in cells expressing US11 ${ }^{\text {Q192L }}$, which indicates that US11 uses its transmembrane region to recruit the class-I heavy chain into a complex with Derlin-1.

Next, the authors created a Derlin-1 construct, in which green fluorescent protein (GFP) was attached to its cytosolic carboxyl terminus. This construct extended the half-life of the class-I heavy chain recruit p97 to Derlin-1. from 5 to 30 minutes in US11-expressing cells, which indicates that, without blocking the binding of the class-I heavy chain to US11 or Derlin-1, the GFP moiety delays the exposure of the class-I heavy chain to the cytosol.

In the second paper, Rapoport and co-workers used a different approach and identified the receptor that links p97 (also known as VCP) to the ER membrane. They actually isolated two proteins in near-stoichiometric amounts - Derlin-1 and a protein that was named VIMP (for VCP-interacting membrane protein).

As VIMP has a large cytosolic domain, the authors proposed that this domain interacts with p97. This proved to be correct, and they showed that this domain recruits $\mathrm{p} 97$ together with its co-factors. Furthermore, using immunofluorescence microscopy, they showed that VIMP seems to

Next, Rapoport and colleagues investigated whether Derlin-1-VIMP is involved in retro-translocation, and they showed that Derlin-1-VIMP interacts with the class-I heavy chain

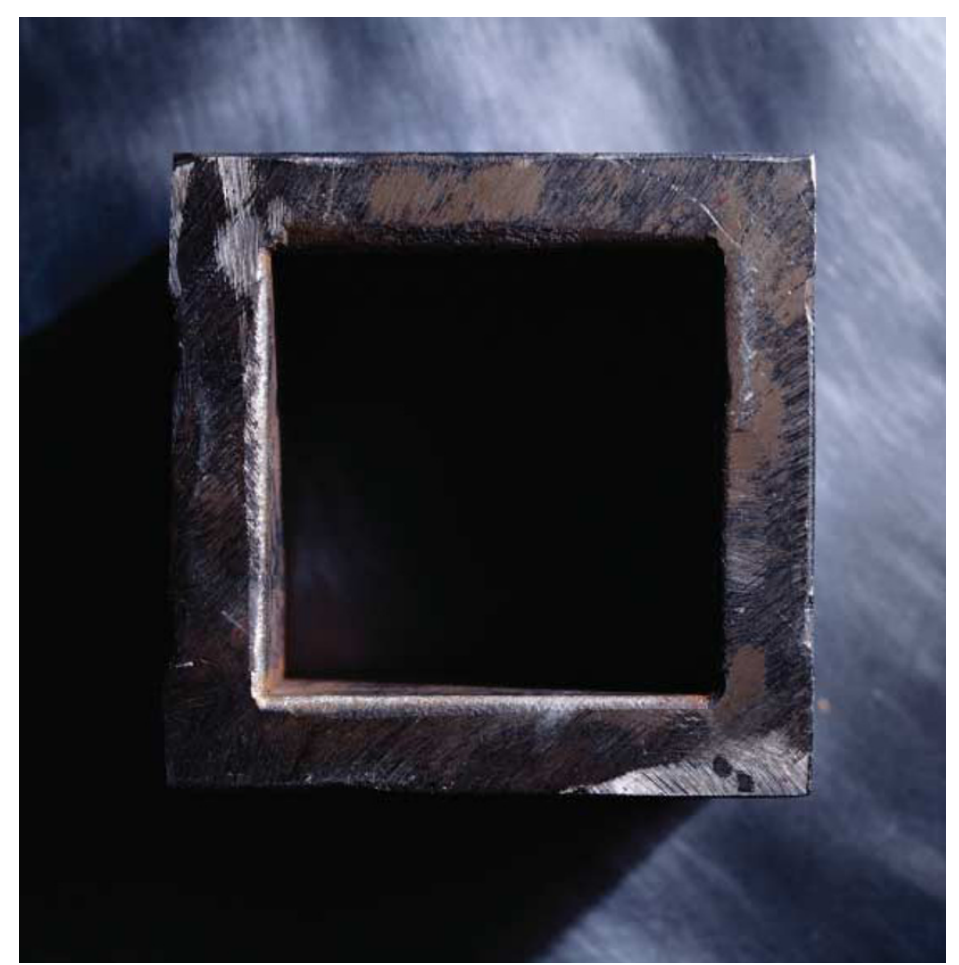
Rapoport and co-workers tested whether the Derlin-1-VIMP complex has a general function in the retrotranslocation of misfolded proteins and their results indicate that it can associate with a large set of substrates. Furthermore, depleting Derlin-1 levels in Caenorhabditis elegans using RNA interference induced ER stress in many cell types.

Together, these groups have therefore identified a putative waste-disposal chute in mammals called Derlin-1. Derlin-1 mediates the retrotranslocation of a subset of misfolded proteins from the ER and, if it can be shown that Derlin-1 does form a channel, it seems only a matter of time before other such retro-translocation channels are identified.

\section{(i) References and links} ORIGINAL RESEARCH PAPERS Lilley, B. N. \& Ploegh, H. L. A membrane protein required for dislocation of misfolded proteins from the ER. Nature 429, 834-840 (2004) | Ye, Y. et al. A membrane protein complex mediates retrotranslocation from the ER lumen into the cytosol. Nature 429, 841-847 (2004)

FURTHER READING Schekman, R. Cell biology: a channel for protein waste. Nature $\mathbf{4 2 9}, 817-818$ (2004)

in US11-expressing cells. In agreement with Lilley and Ploegh, they also noted that an interaction between Derlin-1 and US11 is functionally important for this process.

In the final part of their study, 